\title{
SOCIALITÀ, IDENTITÀ E “DISORDINE” NEI QUARTIERI POPOLARI DEL SECONDO DOPOGUERRA IN ITALIA
}

\author{
Carolina De Falco \\ Università della Campania "Luigi Vanvitelli"
}

ORCID: https://orcid.org/0000-0002-8209-0024

\section{SOMMARIO}

La progettazione dei quartieri popolari del secondo dopoguerra in Italia risente dell'allontanamento dai temi principali del Movimento Moderno: le case in linea parallele, disposte ordinatamente lungo I'asse eliotermico, sono sostituite da gruppi di residenze differenti per tipologia, sfalsate o ruotate fra loro, in modo da ottenere spazi a verde e luoghi urbani per incentivare la socialità. Inoltre, per evitare la conformità, gli edifici presentano varietà nelle aperture e nella texture delle facciate, tanto da caratterizzare il paesaggio urbano, alla ricerca di armonia, bellezza e valori identitari. L'apparente disordine, infatti, è in realtà governato da una regola, che si rivela nel momento in cui il degrado e I'incuria portano a trasgredirla. Nella prospettiva della storia dell'architettura e della città, si propone il risultato di alcuni casi studio in Italia che rendono evidenti tali riflessioni.

Parole chiave: storia della città, quartieri popolari, secondo dopoguerra, INA-Casa, socialità e identità

\section{ABSTRACT}

The design of working-class neighbourhoods in post-war Italy was influenced by a departure from the main themes of the Modern Movement: terraced houses, arranged neatly along the heliothermic axis, were replaced by groups of residences of different types, staggered or rotated in relation to each other to create green and urban spaces that encourage sociability. To avoid uniformity, buildings offered variety in the openings and texture of the facades, so as to characterise the townscape in a quest for harmony, beauty and identity. This apparent disorder was, in actual fact, governed by a rule, which is revealed when degradation and neglect result in it being transgressed. From the perspective of the history of architecture and the city, this paper proposes the results of some cases study in Italy that make these points clear.

Keywords: history of the city, working-class neighbourhoods, post-war architecture, INA-Casa, sociability and identity

La città è certamente il luogo dove si rende maggiormente necessario il tentativo dell'uomo di imporre un ordine che ne renda vivibile gli spazi. La definizione di norme e piani in campo urbanistico è determinate nell'individuare le strategie più opportune a prefigurarlo. In ogni caso, I'ordine non può essere esclusivamente formale, ma deve riguardare anche l'organizzazione sociale, la vita economica, il potere politico, nonché la cultura che esprime il sentire collettivo, in qualsia- si periodo storico. D'altro canto, il disordine non deve essere considerato solo con un'accezione negativa, ma anche come segnale della vitalità sociale di una determinata città e ciò provoca una dialettica dove in realtà ordine e disordine «si oppongono in una specie di lotta senza quartiere, ma pur opponendosi sono elementi dinamici I'uno dell'altro, si sostengono vicendevolmente, non si contrappongono»'. È quanto accade nel caso delle periferie, nate come ampliamento dei 
centri urbani, le quali pur se differenti tra loro per cronologia costruttiva, dislocazione, funzionalità per il territorio, nonché per lo stato del degrado che le caratterizza, «costituiscono un contributo alla particolare forma di ordine di quella determinata città e insieme una continua incentivazione al disordine»².

Esiste inoltre un ulteriore aspetto da considerare, quello percettivo. In particolare, la cultura architettonica dei primi anni Cinquanta, dedicando particolare attenzione al contesto in cui gli edifici si inseriscono, mette in risalto il valore della stratificazione storica. L'apparente disordine determinato da un tessuto urbano sviluppatosi nel tempo può invece risultare armonico proprio per l'accostamento di edifici nuovi ad altri preesistenti ed appartenenti ad epoche diverse e "ciò avvenne in quanto l'artista intuì la propria opera in uno con la natura circostante, la quale, nella fattispecie, era costituita da opere d'arte preesistenti. In tal modo ebbe origine la bellezza di talune piazze o strade, o complessi di edifici, che, nate in epoche lontane I'una dall'altra, costituiscono ormai un tutto inscindibile, al pari di un'opera d'arte» ${ }^{3}$.

Nel secondo dopoguerra, tale consapevolezza genera l'esigenza di ricreare nel progetto dei nuovi quartieri I' "effetto di città vissuta" piuttosto che realizzata in maniera simultanea. Accade allora che ci si allontana da quanto era stato costruito in maniera ordinata in ottemperanza ai dettami del Razionalismo, a partire dagli esempi in Germania. Senza contare che gli edifici funzionalisti e le "stecche" di case orientate lungo I'asse eliotermico avevano già sollevato non poche critiche relative all'eccessiva uniformità tra gli stessi contemporanei. D'altra parte, in Italia, I'avanguardia del Futurismo aveva posto l'accento sull'eterogeneità degli elementi che definiscono la città: edifici, strade, fabbriche, in una visione dinamica che si contrapponeva a quella della cultura classica.

I quartieri popolari dei primi anni Cinquanta evidenziano dunque la ricerca critica di nuovi modelli di aggregazione degli edifici, secondo un accostamento non schematico e in un apparente "disordine", volto alla creazione di scorci e visuali differenti, anche sulla scia degli studi di Kevin Lynch sulle immagini ambientali ${ }^{4}$. Pertanto, se da un lato sono criticati i fondamenti del Movimento Moderno e dello zoning, dall'altro si guarda alle esperienze europee delle città giardino inglesi, dei nuovi quartieri organici svedesi, dei piani olandesi, e alle green belts americane, configurando accesi dibattiti, dove gli aspetti estetici e formali si saldano a quelli etici e sociali. II complesso del Golden Lane realizzato dagli Smithson a Londra rappresenta il tentativo, nel 1951-52, di elaborare nuovi contesti urbani contrapposti alla rigidità geometrica della Ville Radieuse, che trova spazio nei dibattiti del Team $X^{5}$.

Allo stato attuale, nelle città dove spesso più del disordine predomina il caos, dove sempre maggiori sono le differenze, etniche, religiose e culturali, nasce l'esigenza di comprendere non soltanto il significato di ogni luogo, ma anche "le interrelazioni che uniscono tra loro i vari luoghi attraverso catene di significati che entrano profondamente nello spazio e nel tempo, nella concrezione delle configurazioni tridimensionali e nella storia» ${ }^{6}$. Proprio lo sguardo storico, proposto attraverso le riflessioni nate dallo studio di alcuni quartieri popolari nel sud Italia, "sembra quello capace oggi di offrirci la verifica più sicura di qualsiasi ipotesi sulla città; la città è di per se stessa depositaria di storia»?

\section{II "disordine" per il benessere della città "socievole"}

Ludovico Quaroni si esprime in maniera critica sulla fase anteguerra quando «bisognava dare anche all'Italia la siedlung: bisognava rispondere alla richiesta degli architetti che volevano qualcosa di simile, e che in fondo si accontentavano di avere, tutte in fila, tante casette uguali», rendendo in tal modo evidente la ricerca di un modello di aggregazione diverso rispetto al monotono accostamento di unità abitative a schiere parallele, che fosse invece adattabile "caso per caso e luogo per luogo"s.

In questo ambito, diviene determinante I'operato dell'INA-Casa, Ente dedicato alla costruzione delle case popolari in base alla Legge del 1949, nota come Piano Fanfani, in aiuto a coloro che erano ancora costretti a vivere in malsane condizioni igieniche, in baracche o nei rifugi sot-

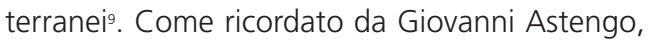
all'INA-Casa sono riconosciuti sia l'azione fonda- 
mentale svolta contro la «disseminazione di case piovute dall'alto» che il merito «di aver rischiarato I'atmosfera dell'edilizia popolare in Italia» ${ }^{10}$. Va sottolineato che anche Bruno Zevi, co-fondatore dell'APAO (Associazione per l'Architettura Organica) e pertanto inizialmente critico nei confronti dell'INA-Casa per la direzione affidata ad Arnaldo Foschini, rappresentante di un linguaggio più vicino al precedente regime, rivede il proprio giudizio". Zevi sostiene infatti che mentre il compito di costruire case popolari era ritenuto «lavoro da geometri, indegno di "artisti" o tecnici qualificati: che ci vuole a progettare una casetta economica? C'è forse bisogno di incomodare architetti?», al contrario l'Ente dell'INA-Casa ha ben compreso che «disegnare un alloggio operaio è arduo e impegnativo. Mobilitando architetti e ingegneri capaci ha elevato lo standard della produzione edilizia» ${ }^{22}$. Ciò è reso possibile anche grazie all'incarico affidato ad Adalberto Libera di dirigere I'ufficio di architettura dell'organo di Gestione dell'INA-Casa, il quale avrà un ruolo importante nella redazione della manualistica elaborata per offrire le linee guida ai progettisti attraverso modelli tipo illustrati, con indicazioni quali, superfici minime complessive, numero dei vani per abitante, sistemazione ottimale di cucina e bagno, fino al trattamento degli esterni' ${ }^{13}$.

A tal proposito, va tuttavia osservato che nelle prescrizioni dell'INA-Casa è raccomandato di fare attenzione alle esigenze dell' «uomo reale e non di un essere astratto, dell'uomo cioè che non ama e non comprende le ripetizioni indefinite e monotone dello stesso tipo di abitazione fra le quali non distingue la propria che per un numero, non ama le sistemazioni a scacchiera, ma gli ambienti raccolti e mossi al tempo stesso» ${ }^{14}$. Se da un lato, quindi, si verifica uno sforzo per mettere ordine nella situazione di emergenza post bellica, dall'altro lato, come sottolineato da Zevi, I'intento è quello di riuscire a soddisfare «qualcosa di più della mera funzionalità, qualcosa di impercettibile [...] che si sente nel viverci: qualcosa che trasforma quattro mura in quattro mura pensate, e pensate affettuosamente, e che in definitiva determina il passaggio dall'edilizia all'architettura» ${ }^{15}$.

Quando all'inizio degli anni Cinquanta, tra i primi quartieri INA-Casa, sono costruiti il Tiburti- no a Roma e La Falchera a Torino, sotto la guida rispettivamente di Ludovico Quaroni con Mario Ridolfi e di Giovanni Astengo, sembra che la strada giusta contro la crescita indifferenziata sia stata finalmente trovata. L'ambizione è quella di realizzare complessi di unità residenziali fondate su un rapporto più consapevole fra l'uomo e la natura, articolate intorno a un "nucleo" con il luogo per l'istruzione e "autosufficienti" grazie ai servizi collettivi, con la consapevolezza del valore educativo, sul piano sociale, fornito da un'ambiente di vita confortevole. Allontanandosi da schemi di tipo seriale, declinati in aggregazioni in linea parallele, i nuovi quartieri sono formati da abitazioni differenti per tipologia: case in linea e case a torre, sfalsate o ruotate fra loro, in modo da ottenere oltretutto spazi a verde destinati alla vita pubblica. L'obiettivo è anche quello di facilitare la reciproca conoscenza tra i residenti, attraverso le cosiddette "unità di vicinato": «unità sociali nelle quali la vita si può svolgere con minori costrizioni, minor peso, più libertà e più ricchezza che non nell'indistinto agglomerato urbano» ${ }^{16}$. Anticipate nel 1929 dai neighborhood unit di Clarence Arthur Perry, in occasione della redazione del Piano Regionale di New York, le unità di vicinato divengono fondamentali nel progetto del quartiere La Martella a Matera elaborato, tra il 1952 e il 1954, da Ludovico Quaroni, con Federico Gorio, Michele Valori, Lugi Agati e Piero Maria Luglip.

A tal proposito, non si può non citare l'esponente italiano del Team X, Giancarlo De Carlo, il quale sottolinea che sono gli individui a dare forma allo spazio e gli usi si sovrappongono rompendo i vincoli con la funzione: la tipologia acquisisce minore importanza, la realtà diversificata, e apparentemente "disordinata", esclude la ripetizione in serie. Secondo De Carlo, non solo nell'ordine c'è la noia frustrante dell'imposizione, mentre il disordine al contrario esprime la fantasia esaltante della partecipazione, quanto quest'ultimo "possiede una sua struttura ramificata e complessa che, non essendo istituzionalizzata, si rinnova di continuo, reinventando a ogni istante le immagini di una realtà che si trasforma» ${ }^{18}$. Pertanto, la giustapposizione di elementi in composizioni chiuse, che aveva caratterizzato la modernità, si trasforma in una combinazione aperta, vitale e 


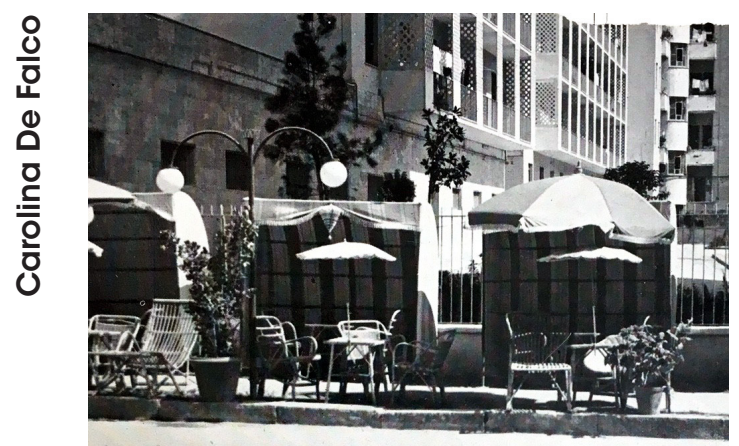

Fig. 1. Napoli, spazio pubblico destinato a bar a Fuorigrotta. Sullo sfondo uno degli edifici di Carlo Cocchia (Da: Cocchia, Carlo. "Aspetti dell'edilizia popolare a Napoli". In Edilizia popolare 17 (1957), 19)

ricca di eventi spaziali, anche simultanei, dove le attività si sovrappongono e si moltiplicano.

Si realizza pertanto una rinnovata cultura dell'abitare, che non coincide più solo con lo spazio privato della residenza: «la città non è fatta solo di addizioni di alloggi. La città è fatta anche di servizi, attrezzature, infrastrutture, spazi vuoti, spazi aperti, giardini, parchi, ecc., e l'abitare avviene nell'insieme di queste attività svariate ${ }^{19}$ Anzi, per evitare il rischio di realizzare complessi a carattere esclusivamente residenziale o "dormitorio", i servizi pubblici e gli spazi collettivi, già essenziali nella vita di una città, «divengono addirittura indispensabili per un quartiere di nuova formazione, senza tradizioni, senza uno spirito, un'anima comune a tutti gli abitanti, che appunto vanno cercando nel centro sociale o nei negozi l'occasione di non sentirsi più soli»»20. D'altro canto, van den Booek e Bakema, autori della prima strada pedonalizzata a uso commerciale in Europa, il Lijnbaan a Rotterdam, realizzata tra il 1948 e il 1953, scompongono il quartiere in blocchi residenziali di altezza e tipologia differente, garantendone l'unità attraverso "gruppi visuali" e servizi sociali21.

Nell'ambito dell'edilizia popolare a Napoli22, incisiva è l'opera di Carlo Cocchia, presidente della Sezione Campana dell'Istituto Nazionale di Urbanistica e docente di Composizione Architettonica al Politecnico di Milano, paragonato dal più giovane De Carlo a Niemeyer «per il rigore e l'asciuttezza del suo linguaggio» ${ }^{23}$. Per quanto riguarda i nuovi quartieri, anche Cocchia racco-

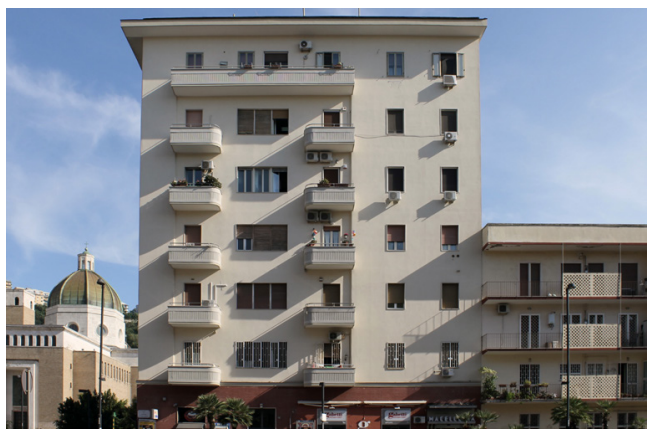

Fig. 2. Napoli, Carlo Cocchia, gruppo di edifici a Fuorigrotta in cui risulta evidente la differenza di tipologia e l'asimmetria delle aperture. I balconi dell'edificio più basso sulla destra presentano un'originale schermatura a fori per nascondere gli stendini (Foto De Falco, 2017)

manda soluzioni spaziali tendenti a prospettare le abitazioni verso l'interno, in modo da «predisporre gli abitanti a più frequenti incontri, favorevoli alla instaurazione di migliori rapporti di vicinato ${ }^{24}$. Infatti, attraverso il quotidiano avvicinamento nelle scuole, negli spazi di gioco, nei negozi, nelle strade interne «intime e circoscritte, simili a passaggi obbligati in entrata della residenza come in uscita, nascono automaticamente quei rapporti scambievoli di interessi comuni e quei sentimenti di solidarietà che costituiscono le premesse della convivenza umana» ${ }^{25}$.

In via Giulio Cesare a Fuorigrotta, tra il 1952 e il 1954, non lontano dai noti edifici a ballatoio realizzati da Luigi Cosenza a Viale Augusto, Cocchia realizza un gruppo di otto palazzi che rende evidente il cambiamento di linguaggio. I primi sono scanditi dalla maglia modulare dei pilotis e declinano con raffinate variazioni il tema del blocco multipiano in linea, eppure, tali «costruzioni logiche» e simmetriche risultano "carenti sotto I'aspetto della morfologia urbana per la programmatica rinuncia a conformare un ambiente spaziale unitario e riconoscibile ${ }^{26}$. Al contrario, gli edifici di Cocchia risultano così diversificati da non sembrare frutto di una progettazione simultanea: quattro sono formati da blocchi sfalsati e prospettano anche su piazza San Vitale, collegati da un corpo basso destinato a negozi allo scopo di «vivificare la strada», gli altri, con altezze variabili da quattro a dieci piani, sono ideati in modo da «disporre più liberamente i corpi di fabbrica, per ottenere risultati assai più concreti degli ef- 


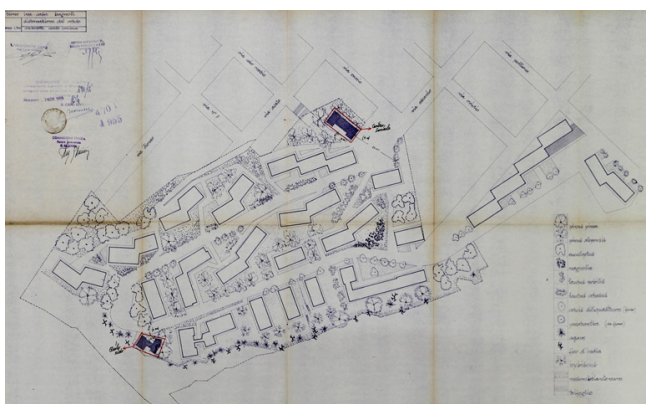

Fig. 3 Napoli, Carlo Cocchia, rione INA-Casa a Bagnoli, planimetria 1/500, 1955 (Archivio Storico Istituto Autonomo Case Popolari Napoli, Vol. "Licenze Edilizie. Rioni Bagnoli ed Agnano")

fetti esteriori, cioè di ambienti espressivi di calore umano e conforto civile» ${ }^{27}$ (fig. 01). II raffinato contrasto del colore rosso mattone sull'intonaco crema dei prospetti e l'ideazione di un'originale schermatura a fori per nascondere gli stendini sui balconi di alcuni edifici lascia trasparire lo straordinario contributo fornito da Cocchia al tema della residenza, ricercato non solo nella costante revisione dei dettami del razionalismo internazionale, avvertiti troppo riduttivi, quanto nella reinterpretazione del senso dell'abitare (fig. 02).

Da tale punto di vista, i complessi residenziali progettati da Cocchia per I'INA-Casa a Bagnoli, a Barra e infine a Secondigliano, cui si aggiunge quello di Stefania Filo Speziale ad Agnano, risultano di particolare interesse in quanto tra i primi a seguire un andamento organico, oltretutto precedenti al più noto quartiere La Loggetta di Giulio de Luca, del 195628. A Bagnoli, nel 1952, Cocchia dispone gli edifici in continuità con le vie preesistenti senza proseguirne però l'impianto a scacchiera, ma anzi rompendone la simmetria e proponendo uno schema di matrice organica, all'interno del quale venticinque edifici si dispongono in maniera irregolare lungo la strada che l'attraversa a monte e ripiega con un'ampia curva alla quota inferiore (fig. 03). Non lontano, sopra un'altura affacciata sull'ex fabbrica dell'Italsider, di fronte a Nisida, il quartiere progettato dalla Filo Speziale, nel 1953, si sviluppa con andamento avvolgente nel rispetto dell'orografia del terreno ${ }^{29}$. Via Tacito conduce in salita al cuore del rione che comprende le strutture collettive con il mercato, il centro sociale e quarantotto edifici residenziali,

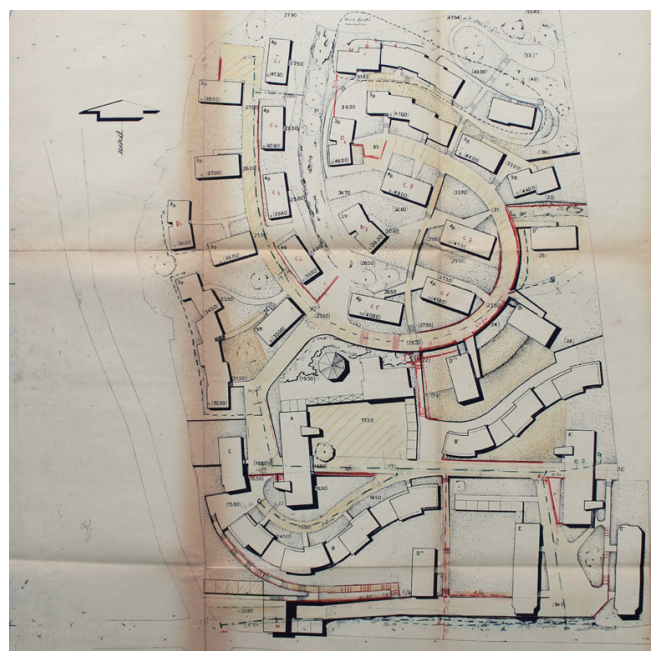

Fig. 4. Napoli, Stefania Filo Speziale, rione INA-Casa ad Agnano, planimetria 1/500, 1953 (Archivio Storico Istituto Autonomo Case Popolari Napoli, Vol. "Licenze Edilizie. Rioni Bagnoli ed Agnano")

di cui la metà affidati al romano Giorgio Costadoni, differenti per tipologia e disposti in maniera variata, tanto che «chi percorre queste vie ha un susseguirsi di sensazioni e di visuali continuamente variate; chi vi abita agevolmente riconosce gli slarghi e si affeziona al suo angolo»30 (fig. 04).

Di particolare interesse è l'intervento di Carlo Cocchia a Barra, che si inserisce nel piano urbanistico di Luigi Cosenza, del 1945-1946, il quale aveva previsto l'ampliamento dell'asse sette-ottocentesco di corso Sirena con i rioni D'Azeglio, da lui stesso progettato, e Cavour, costituiti dalla disposizione "razionale" di un unico tipo di edificio ripetuto in serie parallele. A "rompere" le maglie ordinate del piano Cosenza interviene Cocchia il quale, tra il 1950 e il 1952, progetta per I'INA-Casa il Parco Azzurro, composto da nove edifici bassi e tre case a torre (fig. 05). La distribuzione sfalsata dei palazzi alti consente di ricavare spazi liberi e di animare la zona. Lo sbalzo dei terrazzi collocati a una estremità degli edifici a torre contribuisce nel determinare la spiccata asimmetria dell'insieme. Annoverato, insieme a La Falchera, tra gli esempi migliori mostrati al IV Congresso Nazionale di Urbanistica, tenutosi a Venezia nel 1952 ${ }^{31}$, il quartiere a Barra è segnalato tra i casi all'avanguardia anche sulle pagine della rivista Domus, che gli dedica attenzione al 


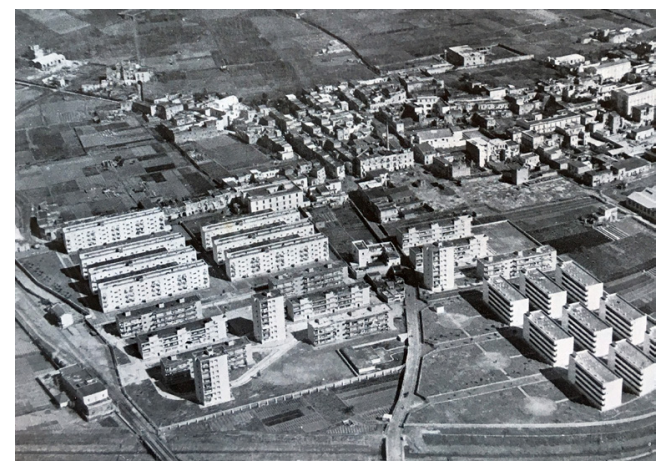

Fig. 5. Napoli, Carlo Cocchia, veduta aerea del rione Parco Azzurro a Barra in primo piano; sulla destra il rione D'Azeglio di Luigi Cosenza e alle spalle il rione Cavour (Da: L'INA-Casa al IV Congresso Nazionale di Urbanistica, Venezia 1952. Roma: Società Grafica Romana, 1953)

pari delle architetture di Mario Ridolfi a Terni e di Mario De Renzi e Saverio Muratori a Valco San Paolo a Roma, riconoscendo che «Napoli ha un'estrema importanza nell'architettura moderna» in Italia32 (fig. 06). Successivamente, tra il 1957 e il 1962, Cocchia realizza anche il quartiere a Secondigliano nel quale trasferisce le riflessioni e le esperienze maturate, tanto da essere segnalato «tra i maggiori e più significativi del secondo settennio INA-Casa» ${ }^{33}$.

\section{Paesaggio urbano: varietà e identità}

Nell'ambito di queste considerazioni, si intende anche riflettere sull'importanza conferita in fase progettuale all'aspetto esteriore degli edifici, strettamente collegata al tema, quanto mai attuale, della vivibilità dei luoghi pubblici. Ancora più singolare è che l'armonia sia stata raggiunta in una fase di contestazione verso l'ordine costituito dalla simmetria e dall'uniformità, in favore della varietà compositiva dell'ambiente urbano, anche se, va sottolineato, comunque sottesa a una regola. D'altra parte, in epoca recente, l'impiego delle geometrie frattale e topologica libera dalla necessità di "semplificare" il mondo attraverso i teoremi euclidei, definendo nuove forme di estetica in cui l'irregolarità non è più percepita come caratteristica negativa. Al contrario, I'odierno disordine dei luoghi urbani è stato in parte determinato dalla noncuranza e dal non aver inteso la qualità dell'originario disegno di progetto, mentre invece le opere d'autore realizzate

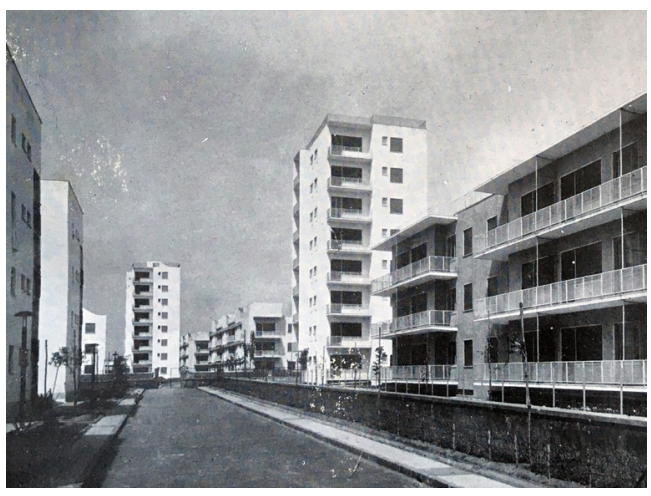

Fig. 6. Napoli, Carlo Cocchia, rione INA-Casa Parco Azzurro a Barra (Da: L'INA-Casa al IV Congresso Nazionale di Urbanistica, Venezia 1952. Roma: Società Grafica Romana, 1953)

andrebbero sottoposte a tutela. Senza contare che un ambiente insediativo nel quale si riesca a creare "bellezza" è anche socialmente più vivibile: il paesaggio urbano crea, infatti, legami tra i suoi abitanti ed esprime un senso condiviso e tacito fra coloro che vi risiedono.

L'auspicio di Bruno Zevi «costo a vano uguale per tutti ma case tutte diverse» è dunque reso possibile, non solo dalla disposizione eterogenea degli edifici tra loro, ma anche dalla diversità con cui sono trattate le facciate ${ }^{34}$. La rotazione dei volumi, gli aggetti dei balconi e i vuoti delle logge, con cui sono realizzate, creano un effetto di movimento e di varietà, affidata inoltre alla scelta accurata dei materiali lapidei a vista, della texture e del colore degli intonaci. I nuovi quartieri sono progettati pertanto nella consapevolezza «dell'importanza dell'aspetto esteriore della casa, del suo affiancarsi ad altre case in mutuo armonico rapporto, del loro collaborare a costituire uno spirito collettivo del rione, della strada, della piazza, dell'ambiente sociale nel quale l'uomo, in Italia specialmente, vive più ancora che nell'interno dell'abitazione» ${ }^{35}$. A tal proposito, va ricordata la fondamentale influenza di Gordon Cullen sul tema del paesaggio urbano ${ }^{36}$, il quale affermava che «un solo edificio è architettura ma due edifici formano Townscape [...] Moltiplicate questo per la dimensione di una città ed avrete l'arte dell'ambiente» ${ }^{37}$.

Questa ricerca d'identità è ciò che rende qualitativamente distinguibile il paesaggio urbano dei quartieri dei primi anni Cinquanta. Non è un 


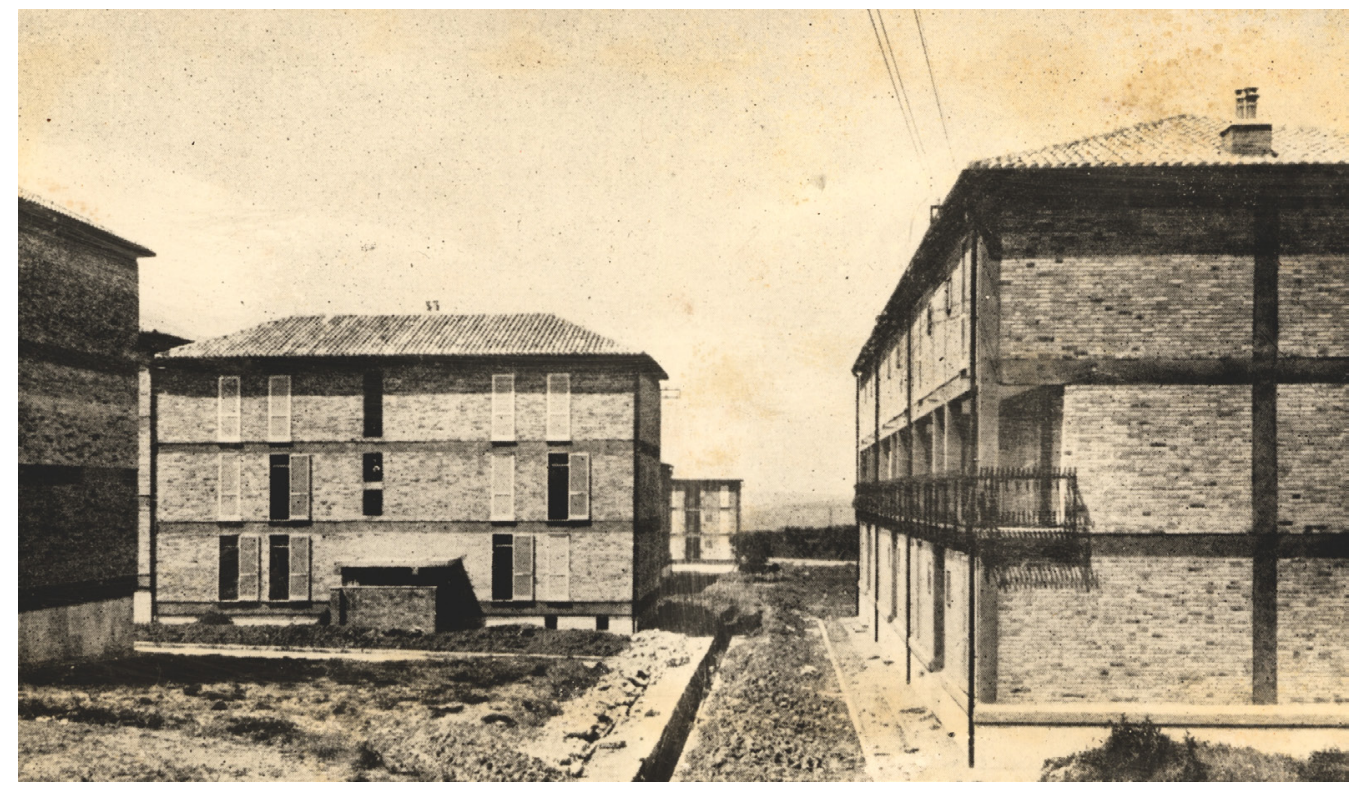

Fig. 7. Matera, quartiere Spine Bianche. Gli edifici di Michele Valori e Federico Gorio e sulla destra quello di Mario Fiorentino e Hilda Selem (MAXXI Museo nazionale delle arti del XXI secolo, Roma. Collezione MAXXI Architettura. Fondo Valori, F42550)

caso se il complesso di case popolari di Giovanni Astengo a Torino, caratterizzato dalle cortine in mattoni degli edifici a tre piani, sia noto come La Falchera Vecchia per differenziarlo, anche "storicamente", dalla Falchera Nuova, costruita negli anni Settanta. Dello stesso autore, il complesso in via dei Filosofi a Perugia presenta "giardini rigogliosi, tetti in coppi, scalinate piene di vita, stradine prive di traffico, muri in mattoni», tanto che il fotografo Guido Guidi nel reportage svolto in occasione del cinquantesimo anniversario dell'istituzione del piano INA-Casa, occupa «ormai da due ore lo stesso piazzale senza smettere mai di trovare nuovi spunti per il suo lavoro» ${ }^{38}$.

Va sottolineato che tale "pluralità nell'unità" del piano urbanistico di quartiere è raggiunta anche dall'avere, quasi sempre, affidato i progetti architettonici degli edifici a più gruppi, spesso vincitori ex aequo dei concorsi, a loro volta composti da numerosi professionisti, architetti e ingegneri, anche di formazione diversa, la cui interazione provoca una straordinaria circolazione di idee. È il caso a Napoli dei quartieri di San Giovanni a Teduccio e di Canzanella a Soccavo, nei quali intervengono architetti di estrazione romana. Nel primo caso, Carlo Aymonino, Carlo Chiarini,
Marcello Girelli e Sergio Lenci, si ritroveranno successivamente a lavorare anche nel quartiere Spine Bianche a Matera, formidabile cantiere sul quale si concentra l'attenzione della cultura italiana di quegli anni; nel secondo caso, viceversa, Mario Fiorentino dopo l'intervento materano porterà la propria esperienza a Canzanella ${ }^{39}$.

A Spine Bianche, da un lato con il noto "edificio per abitazioni e negozi" De Carlo "tradisce" i principi del Movimento Moderno, con la scelta di un linguaggio più aderente alla realtà locale, e rimarca l'importanza dell'attività commerciale nella vita del quartiere, dall'altro i diversi gruppi di edifici danno prova di autonomia formale, pure nell'ambito di un disegno fortemente unitario, sia a livello urbanistico, ma anche nell'impiego uniforme del rivestimento esterno in mattoni a faccia a vista, di produzione locale, dall'effetto vibrante nelle tonalità dal giallo al rosa.

A titolo di esempio tra gli altri, gli edifici realizzati da Mario Fiorentino con Hilda Selem -corrispondente di The Architectural Review all'epoca della vicedirezione di Cullen- introducono elementi di novità nei balconi coperti al primo piano, corredati da ringhiere in ferro sagomate per accogliere vasi da fiori, ricavati da un arretramento 


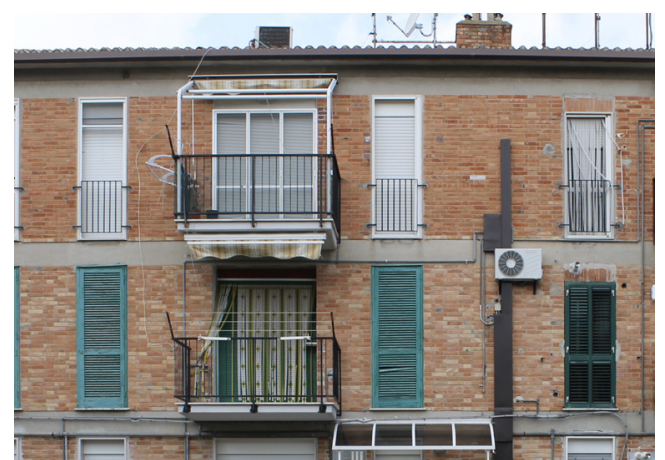

Fig. 8. Matera, quartiere Spine Bianche. Un esempio di alterazione delle aperture sulla facciata di un edificio (Foto De Falco, 2019)

del filo della facciata che provoca in tal modo lo svuotamento degli angoli.

Un altro effetto di varietà, come nel caso degli edifici progettati da Michele Valori e Federico Gorio, è affidato alle aperture con persiane lignee tinteggiate con colori differenti e caratterizzate da un'anta unica da solaio a solaio, la quale inoltre determina, se aperta o se chiusa, un gioco di asimmetrie che richiama il palazzo Borsalino ad Alessandria di Ignazio Gardella, sebbene con una tecnologia di tipo tradizionale a cerniera (fig. 07). Invece, purtroppo, il disordine è provocato dall'abusivismo che ha condotto alla trasformazione casuale e individuale delle aperture, conservate oramai solo in minima parte allo stato originario, dovuta o alla sostituzione delle persiane con le tapparelle o al ribassamento della luce e all'inserimento di chiusure a doppia anta (fig. 08).

L'attenzione all'aspetto e all'identità che ha improntato i progetti dei primi quartieri del dopoguerra è venuta invece a mancare successivamente, per lasciare il posto a interventi di speculazione, destinando le periferie a luoghi anonimi, regni del disordine 0 "non luoghi"40. La sensibilità verso la tutela dell'architettura moderna sta iniziando a concretizzarsi solo di recente ${ }^{41}$. La consapevolezza del bene pubblico è fondamentale per favorire «le opportunità di una polis amica e più socievole contro la solitudine del cittadino globale» ${ }^{42}$.

Il rapporto tra spazio costruito e spazio aperto è dunque cruciale per creare luoghi abitabili e costituisce una componente innovativa all'interno del panorama dell'edilizia economica e popolare degli anni Cinquanta. Pertanto, è necessario un approccio che miri al recupero della dimensione umana della vita in comunità, oggi quanto mai attuale, a partire dal patrimonio urbano, con l'inclusione dei quartieri più periferici, rintracciandone e tutelandone i valori identitari, per rendere i luoghi pubblici più "ordinati" e vivibili. 
NOTE

Indovina, Francesco. 2017. Ordine e disordine nella città contemporanea. Milano: Franco Angeli, 11. Vedi anche Di Biagi, Paola and Gabellini, Patrizia, ed. 1992. Urbanisti italiani: Piccinato, Marconi, Samonà, Quaroni, De Carlo, Astengo, Campos Venuti. Roma: Laterza.

2 Indovina, Francesco. 2017. Ordine e disordine nella città contemporanea. Milano: Franco Angeli, 135. Vedi anche Oriol Nel·lo, Dell'ordine e del disordine urbano, 7 dicembre 2018. Accessed July, 10, 2020 https://www.casadellacultura.it/832/dell-ordine-e-del-disordine-urbano

Su Indovina vedi anche Belli, Gemma. 2016. A colloquio con I'urbanistica italiana. Per la storia di una nuova tradizione. Napoli: Clean.

3 Cocchia, Carlo. 1950. Strutture e spazi nella natura. Napoli: Pellerano Del Gaudio, 17.

4 Andriello, Vincenzo. La città vista attraverso gli occhi degli «altri». Lynch, The Image of the City, 1960. In Di Biagi, Paola, ed. 2009. I classici dell'urbanistica moderna. Roma: Donzelli, 145-161.

5 Biraghi, Marco. 2008. Storia dell'architettura contemporanea 19452008. Vol. II. Torino: Einaudi, 123-124.

6 De Carlo, Giancarlo. 1989. "L'interesse per la città fisica". Urbanistica, 95, 17.

7 Rossi, Aldo. 2011 (I ed. 1966). L'architettura della città. Macerata: Quodlibet, 144. In questa prospettiva vedi De Falco, Carolina. 2018. Case INA e luoghi urbani. Storie dell'espansione occidentale di Napoli. Napoli: Clean.

8 Quaroni, Ludovico. 1957. "La politica del Quartiere". In Urbanistica, 22,6 .

Di Biagi, Paola ed. 2001. La grande ricostruzione. II piano Ina-Casa e I'Italia degli anni '50. Roma: Donzelli; vedi anche Caramellino, Gaia and Sotgia, Alice ed. 2014. "Tra pubblico e privato. Case per dipendenti nell'Italia del secondo Novecento". In Città \& Storia, IX, 319-327.

${ }^{10}$ Astengo, Giovanni. 1951. "Nuovi quartieri in Italia", In Urbanistica, 7, 9. Sulla figura di Astengo si rinvia in breve a Zucconi, Guido. 2003. Astengo Giovanni. In Olmo, Carlo ed. Dizionario dell'Architettura del XX secolo, vol. I, Roma: Istituto della Enciclopedia Italiana, 106-109 e di recente Ciacci, Leonardo, Dolcetta, Bruno and Marin, Alessandra. 2009. Giovanni Astengo. Urbanista Militante. Venezia: Marsilio.

11 La vicenda del rapporto tra architettura e politica è analizzato da Zeier Pilat, Stephanie. 2019. Ricostruire I'Italia. I quartieri Ina.Casa del dopoguerra. Roma: Castelvecchi.

12 Zevi, Bruno. 1970. "La DC attacca I'Ina-casa. S'impenna saltando I'ostacolo quartiere città". In Cronache di architettura. 174, 447-448.

13 INA-Casa. 1949. Piano incremento occupazione operaia. Case per lavoratori. Suggerimenti, Norme e schemi per l'elaborazione e presentazione dei progetti. Fascicolo 1. Roma: Istituto Poligrafico dello Stato. INA-Casa. 1950. Suggerimenti, esempi e norme per la progettazione urbanistica. Progetti tipo. Fascicolo 2. Roma: Istituto Poligrafico dello Stato. Vedi anche Beretta Anguissola, Luigi ed. 1963. I 14 anni del piano INA-Casa. Roma: Staderini.

14 INA-Casa. 1949. Piano incremento occupazione operaia. Case per lavoratori. Suggerimenti, Norme e schemi per l'elaborazione e presentazione dei progetti. Fascicolo 1. Roma: Istituto Poligrafico dello Stato, 10-11.

15 Zevi, Bruno. 1953. L'architettura dell'INA Casa. In L'INA-Casa al IV Congresso Nazionale di Urbanistica, Venezia 1952. Roma: Società Grafica Romana, 16.

16 Astengo, Giovanni. 1951. "Nuovi quartieri in Italia", In Urbanistica, 7, 9.

17 Mumford, Lewis. 1954. "The Neighboorhood and the Neighborhood unit", in Town Planning Review, January, 256-270; Caves, Roger W. 2004. Encyclopedia of the City. London: Routledge, 513; Lenza, Cettina. 2019. Paesaggi urbani del Novecento: i borghi e i quartieri di Matera tra diritto all'abitare e diritto alla bellezza. In Matera, città del sistema ecologico uomo/società/ natura: il ruolo della cultura per la rigenerazione del sistema urbano/territoriale. Girard, Luigi F. and Trillo, Claudia and Bosone, Martina ed. Napoli: Giannini, 139-161.
18 De Carlo, Giancarlo. 1970. "II pubblico dell'architettura". In Parametro, n. 5, 11. Vedi anche De Carlo, Giancarlo. 1972. "Ordine-istituzione educazione-disordine". In Casabella, n. 368-369, 65-71; Blake Peter and De Carlo, Giancarlo and Richards, Jim M. 1973. L'architettura degli anni Settanta. Milano: Saggiatore. Sulle opere di De Carlo vedi da ultimo il catalogo della mostra: Guccione, Margherita and Vittorini, Alessandra ed. 2005. Giancarlo De Carlo. Le ragioni dell'architettura. Milano: Electa.

19 Bunčuga, Franco. 2000. Conversazioni con Giancarlo De Carlo. Architettura e libertà. Milano: Eleuthera, 146.

20 Quaroni, Ludovico. 1957. "La politica del Quartiere". 1957. In Urbanistica, 22, 10.

21 Tafuri, Manfredo. 1988. II panorama internazionale negli anni '50 e '60. In Tafuri, Manfredo and Dal Co, Francesco. Architettura contemporanea. Milano: Electa, 332.

22 Sull'edilizia popolare a Napoli, con un approccio urbanistico, vedi: Stenti, Sergio 1993. Napoli moderna, città e case popolari 1868-1980. Napoli: Clean; Carughi, Ugo, ed. 2006. Città Architettura Edilizia pubblica. Napoli e il Piano INA Casa. Napoli: Clean; Pagano, Lilia. 2012. Periferie di Napoli. Roma: Aracne.

${ }^{23}$ Caterina, Gabriella and Nunziata, Massimo ed. 1987. Carlo Cocchia cinquant'anni di architettura 19371987. Genova: Sagep, 37.

${ }^{24}$ Cocchia, Carlo. 1961. L'edilizia a Napoli dal 1918 al 1958. Napoli: Società pel Risanamento, 78.

25 Ivi, 79.

${ }^{26}$ Gravagnuolo, Benedetto. 2006. Poeticamente abita I'uomo.... In Buccaro, Alfredo and Mainini, Giuseppe ed. Luigi Cosenza oggi: 1905-2005. Napoli: Clean, 119.

27 Cocchia, Carlo. 1961. L'edilizia a Napoli dal 1918 al 1958. Napoli: Società pel Risanamento, 80. Vedi anche Cocchia, Carlo. 1957. "Aspetti dell'edilizia popolare a Napoli". In Edilizia popolare, n. 17, 19-23.

28 Per un approfondimento su questi quartieri vedi: De Falco, Carolina. 
2018. Case INA e luoghi urbani. Storie dell'espansione occidentale di Napoli. Napoli: Clean.

${ }^{29}$ Ivi, 25 in nota, per ulteriori riferimenti bibliografici sulla Filo Speziale.

30 Astengo, Giovanni. 1951. "Nuovi quartieri in Italia", In Urbanistica, 7, 11.

31 L'INA-Casa al IV Congresso Nazionale di Urbanistica, Venezia 1952. Roma: Società Grafica Romana, 19798.

32 Ponti, Gio. 1952. "Sequenze di paesaggi architettonici". In Domus, 270, 6 .

33 "Il quartiere di Secondigliano di C. Cocchia". 1953. In Casabella continuità, XXIII, 231, 53.

34 Zevi, Bruno. 1953. L'architettura dell'INA Casa. In L'INA-Casa al IV Congresso Nazionale di Urbanistica, Venezia 1952. Roma: Società Grafica Romana, 16.

35 Muratori, Saverio. 1951. "La gestione Ina-Casa e I'edilizia popolare in Italia". In Rassegna critica di architettura, 20-21, 19 e 24.

36 Sull'argomento vedi De Falco, Carolina. 2019. " «Sequenze di paesaggi architettonici»: la costruzione delle case popolari nei primi anni Cinquanta tra Napoli e la Basilicata". ArcHistoR, $12,136-173$, cui si rinvia per ulteriori approfondimenti. Inoltre, sono stata chairman (con G. Belli) della sessione Paesaggi urbani in età moderna e contemporanea al IX Convegno dell'AISU La città globale. La condizione urbana come fenomeno pervasivo. Bologna, 2019/09/11-14, i cui atti sono in corso di stampa.

37 Cullen, Gordon.1953. "Prairie planning in the New Towns". In The Architectural Review, CXIV, 679, 33; qui si cita la traduzione di Andriello, Vincenzo. 1959. Il Townscape. Concetto, limiti, caratteristiche. Roma: Tip. DAPCo, 10. Sull'origine del concetto di paesaggio urbano vedi anche Maderuelo, Javier. 2005. El paisaje. Génesis de un concepto. Madrid: Abada.

38 Marino, Gianluca. 1999. Note da un diario di viaggio con Guido Guidi. In Di Biagi, Paola ed. Guido Guidi. Sequenze di paesaggi urbani. Un itinerario tra quartieri InaCasa, Rubiera: Linea di confine Editore, 10-11; Bori, Sergio. Perugia. Complesso residenziale di via dei Filosofi (Giovanni Astengo, 1956-1962). 2012. In Belardi, Paolo and Menchetelli Valeria ed. Da case popolari a case sperimentali. Un secolo di architettura nell'edilizia residenziale pubblica della provincia di Perugia. Perugia: Fabrizio Fabbri editore, 131

39 Per approfondire vedi De Falco, Carolina. 2019. " "Sequenze di paesaggi architettonici»: la costruzione delle case popolari nei primi anni Cinquanta tra Napoli e la Basilicata". ArcHistoR, 12, 161-169.

40 Augè, Marc. 1992. Non-leux. Introduction à una antropologie de la surmodernité; trad it. Nonluoghi. Introduzione a una antropologia delle surmodernità. Milano: Elèuthera.

${ }^{41}$ Carughi, Ugo and Visone, Massimo ed. 2017. Time Frames: Conservation Policies for Twentieth-Century Architectural Heritage. London: Routledge; Canella, Gentucca and Mellano, Paolo ed. 2019. Architettura d'autore del secondo Novecento. II diritto alla tutela. Milano: Franco Angeli.

42 Treu, Maria Cristina ed. 2015. Per una città socievole. Le alterne fortune di piani e progetti. Novellara (RE): Palazzo Bonaretti Editore, 19. Vedi anche Scandurra, Enzo. 2007. Un paese ci vuole. Ripartire dai luoghi. Troina: Città Aperta. 


\section{REFERENCIAS}

Andriello, Vincenzo. /l Townscape. Concetto, limiti, caratteristiche. Roma: Tip. DAPCo, 1959.

Andriello, Vincenzo. La città vista attraverso gli occhi degli «altri». Lynch, The Image of the City, 1960. In I classici dell'urbanistica moderna, Paola Di Biagi, ed., 145-161. Roma: Donzelli, 2009.

Astengo, Giovanni. "Nuovi quartieri in Italia." Urbanistica 7 (1951): 9-41.

Augè, Marc. Non-lieux. Introduction à una antropologie de la surmodernité. Trad it. Nonluoghi. Introduzione a una antropologia delle surmodernità. Milano: Elèuthera, 1992.

Belli, Gemma. A colloquio con I'urbanistica italiana. Per la storia di una nuova tradizione. Napoli: Clean, 2016.

Beretta Anguissola, Luigi, ed. I 14 anni del piano INA-Casa. Roma: Staderini, 1963.

Biraghi, Marco. Storia dell'architettura contemporanea 1945-2008. Vol. II. Torino: Einaudi, 2008.

Blake, Peter, De Carlo, Giancarlo, and Jim M. Richards. L'architettura degli anni Settanta. Milano: Saggiatore, 1973.

Bori, Sergio Perugia. "Complesso residenziale di via dei Filosofi (Giovanni Astengo, 19561962)." In Da case popolari a case sperimentali. Un secolo di architettura nell'edilizia residenziale pubblica della provincia di Perugia, Paolo Belardi and Valeria Menchetelli, eds., 131. Perugia: Fabrizio Fabbri editore, 2012.

Bunčuga, Franco. Conversazioni con Giancarlo De Carlo. Architettura e libertà. Milano: Eleuthera, 2000.

Canella, Gentucca, and Paolo Mellano, eds. Architettura d'autore del secondo Novecento. II diritto alla tutela. Milano: Franco Angeli, 2019.

Caramellino, Gaia, and Alice Sotgia, eds. "Tra pubblico e privato. Case per dipendenti nell'Italia del secondo Novecento." In Città \& Storia IX (2014): 319-327.

Carughi, Ugo, and Massimo Visone, eds. Time Frames: Conservation Policies for Twentieth-Century Architectural Herita- ge. London: Routledge, 2017. https://doi. org/10.4324/9781315269863

Carughi, Ugo, ed. Città Architettura Edilizia pubblica. Napoli e il Piano INA Casa. Napoli: Clean, 2006.

Caterina, Gabriella, and Massimo Nunziata, eds. Carlo Cocchia cinquant'anni di architettura 1937-1987. Genova: Sagep, 1987.

Caves, Roger W. Encyclopedia of the City. London: Routledge, 2004.

Ciacci, Leonardo, Dolcetta, Bruno, and Alessandra Marin. Giovanni Astengo. Urbanista Militante. Venezia: Marsilio, 2009.

Cocchia, Carlo. Strutture e spazi nella natura. Napoli: Pellerano Del Gaudio, 1950.

Cocchia, Carlo. "Aspetti dell'edilizia popolare a Napoli". In Edilizia popolare 17 (1957), 19-23.

Cocchia, Carlo. L'edilizia a Napoli dal 1918 al 1958. Napoli: Società pel Risanamento, 1961.

Cullen, Gordon. "Prairie planning in the New Towns." The Architectural Review CXIV, $\mathrm{n}^{\circ}$ 679 (1953): 33-36.

De Carlo, Giancarlo. "Il pubblico dell'architettura." Parametro 5 (1970): 4-12..

De Carlo, Giancarlo. "Ordine-istituzione educazione-disordine." Casabella 368-369 (1972): 65-71.

De Carlo, Giancarlo. "L'interesse per la città fisica." Urbanistica 95 (1989): 15-18.

De Falco, Carolina. Case INA e luoghi urbani. Storie dell'espansione occidentale di Napoli. Napoli: Clean, 2018.

De Falco, Carolina. " «Sequenze di paesaggi architettonici»: la costruzione delle case popolari nei primi anni Cinquanta tra Napoli e la Basilicata." ArcHistoR 12 (2019): 136-173.

Di Biagi, Paola, and Patrizia Gabellini, eds. Urbanisti italiani: Piccinato, Marconi, Samonà, Quaroni, De Carlo, Astengo, Campos Venuti. Roma: Laterza, 1992.

Di Biagi, Paola, ed. La grande ricostruzione. II piano Ina-Casa e l'Italia degli anni '50. Roma: Donzelli, 2001. 
Socialità, identità e "disordine" nei quartieri popolari del secondo dopoguerra in Italia

Gravagnuolo, Benedetto. "Poeticamente abita I'uomo...." In Luigi Cosenza oggi: 1905-2005 Alfredo Buccaro and Giuseppe Mainini, eds., 118-121. Napoli: Clean, 2006.

Guccione, Margherita, and Alessandra Vittorini, ed. Giancarlo De Carlo. Le ragioni dell'architettura. Milano: Electa, 2005.

INA-Casa. Piano incremento occupazione operaia. Case per lavoratori. Suggerimenti, Norme e schemi per l'elaborazione e presentazione dei progetti. Fascicolo 1. Roma: Istituto Poligrafico dello Stato, 1949.

INA-Casa. Suggerimenti, esempi e norme per la progettazione urbanistica. Progetti tipo. 1950. Fascicolo II. Roma: Istituto Poligrafico dello Stato, 1950.

"Il quartiere di Secondigliano di C. Cocchia." Casabella continuità XXIII, nº 231 (1953): 53.

Indovina, Francesco. Ordine e disordine nella città contemporanea. Milano: Franco Angeli, 2017.

L'INA-Casa al IV Congresso Nazionale di Urbanistica, Venezia. Roma: Società Grafica Romana, 1952.

Lenza, Cettina. "Paesaggi urbani del Novecento: i borghi e i quartieri di Matera tra diritto all'abitare e diritto alla bellezza." In Matera, città del sistema ecologico uomo/società/natura: il ruolo della cultura per la rigenerazione del sistema urbano/territoriale, Luigi Girard, Claudia F. Trillo, Martina Bosone, eds., 139161. Napoli: Giannini, 2019.

Maderuelo, Javier. El paisaje. Génesis de un concepto. Madrid: Abada, 2005.

Marino, Gianluca. "Note da un diario di viaggio con Guido Guidi." In Guido Guidi. Sequenze di paesaggi urbani. Un itinerario tra quartieri InaCasa, Paola Di Biagi, ed., 10-11. Rubiera: Linea di confine Editore, 1999.

Mumford, Lewis. "The Neighboorhood and the Neighborhood unit." Town Planning Review, (January, 1954): 256-270. https://doi. org/10.3828/tpr.24.4.d4r60h470713003w
Muratori, Saverio. "La gestione Ina-Casa e I'edilizia popolare in Italia." Rassegna critica di architettura 20-21 (1951): 19-24.

Oriol Nel·lo, Dell'ordine e del disordine urbano, 7 dicembre 2018. Accessed July, 10, 2020 https://www.casadellacultura.it/832/dell-ordine-e-del-disordine-urbano

Pagano, Lilia. Periferie di Napoli. Roma: Aracne, 2012.

Ponti, Gio. "Sequenze di paesaggi architettonici." Domus 270 (1952): 1-8.

Quaroni, Ludovico. "La politica del Quartiere." Urbanistica 22 (1957): 4-14.

Rossi, Aldo. L'architettura della città. Macerata: Quodlibet, 2011 (I ed. 1966).

Scandurra, Enzo. Un paese ci vuole. Ripartire dai luoghi. Troina: Città Aperta, 2007.

Stenti, Sergio Napoli moderna, città e case popolari 1868-1980. Napoli: Clean, 1993.

Tafuri, Manfredo. "Il panorama internazionale negli anni '50 e '60." In Architettura contemporanea. Manfredo Tafuri and Francesco Dal Co. Milano: Electa, 1988.

Treu, Maria Cristina, ed. Per una città socievole. Le alterne fortune di piani e progetti. Novellara (RE): Palazzo Bonaretti Editore, 2015.

Zeier Pilat, Stephanie. Ricostruire I'Italia. I quartieri Ina. Casa del dopoguerra. Roma: Castelvecchi, 2019.

Zevi, Bruno. "L'architettura dell'INA Casa." In L'INA-Casa al IV Congresso Nazionale di Urbanistica, Venezia 1952. Roma: Società Grafica Romana, 1953.

Zevi, Bruno. "La DC attacca I'Ina-casa. S'impenna saltando I'ostacolo quartiere città." Cronache di architettura 174 (1970): 447-448.

Zucconi, Guido. "Astengo Giovanni." In Dizionario dell'Architettura del XX secolo, Carlo Olmo, ed. vol. I, 106-109. Roma: Istituto della Enciclopedia Italiana, 2003. 